\title{
Los docentes, los estudiantes y las NTIC: una nueva visión del uso de las tecnologías en el aula de clases
}

\author{
Julián David Cangrejo Patarroyo \\ Estudiante de Lengua castellana \\ Universidad Surcolombiana, Colombia \\ julianchid_12@hotmail.com
}

Las Nuevas Tecnologías de la Información y la Comunicación (NTIC) han logrado que las personas se vean enfrentadas a la cultura revolucionaria del internet, visto como el eje principal de la comunicación del siglo actual, y sean puestas a prueba, desafiadas, para lograr adaptarse a los cambios acelerados de la sociedad. A partir de estos cambios, la institución educativa $\mathrm{y}$ en particular las aulas escolares, han sido empujadas a un espacio tecnológico que domina el mundo actual. Es por eso que los docentes y en cierta forma los estudiantes, han tratado de encontrar la forma de integrar en los procesos de aprendizaje, las herramientas ofrecidas por las NTIC. Los gobiernos -y sus ministerios de educación- han entendido que la incursión de las tecnologías en las escuelas es necesaria, tanto por las nuevas competencias comunicativas, como para alcanzar los estándares actualizados impuestos por la globalización que día a día es acogida en el mundo entero.

La apropiación de las tecnologías de la comunicación en las aulas de clase se ha hecho de manera paulatina y con cierta resistencia por parte de un sector, aún representativo de la comunidad docente, que no logra integrar las NTIC a su ejercicio pedagógico. Es más, desestimula los nuevos avances en telefonía celular, internet, juegos y programas de computador, redes sociales tal vez porque sienten nostalgia de los viejos tiempos y se niegan a encarar lo que para ellos puede ser visto como un reto impuesto.

Si bien es cierto que anualmente los gobiernos de todos los países del mundo invierten millones en el desarrollo de planes y programas de educación que se adecuen al contexto y facilite el aprendizaje no siempre las redes de las comunicaciones se convierten en prioridad y solo las iniciativas personales de los estudiantes nacidos en el nuevo siglo, los nativos digitales son los que de alguna manera se identifican y exigen que las NTIC se integren a los procesos de enseñanza-aprendizaje (Barreras, 2016).

Y no se trata solo de contextualizarse, ponerse a tono con la tecnología. Se trata de consolidar la competencia comunicativa en los procesos de aprendizaje, entendiendo que se aprende y se comunica en lengua.

Una de los rasgos de todas las lenguas del mundo y quizás uno de los más importantes, es la creación y/o formación de nuevas palabras. El español ${ }^{1}$, al igual que otros idiomas, ha tenido que adaptarse a las rápidas olas de cambio y

1 Una manera de hacer más interesante el problema neológico se encuentra en el blog neológico del CVC: El Observatori de Neologia del grupo IULATERM de la Universitat Pompeu Fabra y el Instituto Cervantes por medio de su Centro Virtual lanzaron en octubre de 2015 conjuntamente esta iniciativa de divulgación lingüística. Cada martes desde entonces se difunde un texto con el análisis de un neologismo del español, entendiendo por neologismo aquella palabra que se encuentra documentada en el uso de los hablantes (especialmente en textos de prensa) y que no se encuentra recogida en el diccionario de la Real Academia Española. Para cada neologismo se ofrece información gramatical (categoría, tipo de formación), semántica (ejemplos de uso, explicación del significado) y de otros tipos (origen etimológico, frecuencia de aparición, presencia en obras lexicográficas, etc.) http://blogscvc.cervantes.es/martes-neologico/inicio/ 
ha incrementado el nivel de innovación léxica. Los sectores que más demandan innovación son los de economía e informática. Es más, la comunidad hispanohablante se ha visto obligada a acudir a los préstamos lingüísticos en esta área: browser (navegador), bookmark (marcador), host (computadora anfitriona), link (enlace), password (contraseña), etc., y los jóvenes entre los ocho y los diecisiete años, considerados popularmente de la Generación Z, han liderado los procesos de actualización y préstamo, respondiendo a sus necesidades de comunicación.

Losniñosyjóvenes comprenden perfectamente esto y crean nuevos códigos de comunicación que respondan a lo que quieran expresar. Para dar un ejemplo concreto, en la red social Twitter, las publicaciones están reducidas a 143 caracteres. Los usuarios han tenido que encontrar métodos para lograr que su mensaje se dé, usando abreviaciones o simples juegos que sonoridad

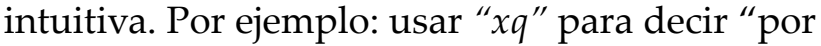
qué" o "porque" dependiendo el caso, " $b n$ " para decir "bien" o "bueno", "fds" usando las siglas para decir "fin de semana" sin mayúscula ni punto, entre otros, todo con el objetivo de ahorrar caracteres y evitar la interrupción del mensaje a expresar. Evidentemente aquí el contexto del mensaje es de suma importancia.

En los centros educativos, los niños y jóvenes han acogido de forma amena a las tecnologías del internet, y con ellas a las redes sociales más populares, como Youtube, Whatsapp, Instagram, Facebook, Twitter, Tumblr, Google y otras, bien para hacer sus labores académicas, o para comunicarse de forma rápida y efectiva con su familia y allegados, y desde luego para divertirse.

En los espacios educativos el internet y las redes sociales también han jugado un papel importante, pues se presentan con la imagen de facilitador de información inmediata y certera. Pero el problema para los docentes surge desde los novedosos e intempestivos cambios en la escritura $^{2}$. Quizá el caso de Twitter, pueda ser de entendimiento lógico, debido al limitante, pero en otras redes sociales que no denotan un límite escritural puede resultar más agresivo. Los constantes errores ortográficos en las publicaciones son permitidos, salvo por un buen autocorrector de teclado o un buen conocimiento de la gramática del idioma. Muchas veces los errores son excusados por "urgencia de inmediatez" o "por desconocimiento del error"; pero no dejan de ser errores. Este tipo de consecuencias se asumen en la escuela, donde el docente se encarga de velar por que sus estudiantes adquieran un buen conocimiento de la gramática y la ortografía de las palabras del español.

Ahora habría que entender la polémica de los neologismos en la web. Entendemos que los neologismos son palabras que se forman, bien sea uniendo palabras con otras, o simplemente creando nuevas palabras con una sonoridad particular, que respondan a una circunstancia necesaria. Muchas veces estas palabras nacen a partir de una raíz definida, un derivado de otro idioma o una intervención onomatopéyica. Con la aparición de las redes sociales, los neologismos suelen tener su nacimiento de la misma forma. Por ejemplo: "befa(o)" es un neologismo que nace precisamente de la sonorización de las siglas de la frase inglesa "best friend for ever" (bffe) que en español significa "mejores amigas(os) por siempre"; es usado sobre todo entre los jóvenes para referirse a una persona especial, un amigo de mucha cercanía. Otro caso más aceptado por la sociedad colombiana es la palabra "parce" o "parcero"; no se sabe con exactitud de dónde proviene la palabra, pero se cree que es descendiente de la palabra portuguesa "parceiro", que en español significa "amigo" o "colega", siendo este el mismo significado en el contexto colombiano. Otro ejemplo importante es el verbo "chatear", proveniente del anglicismo "chat" que en español significa "hablar", "conversar"; es usado comúnmente por los jóvenes para hacer referencia a la interacción y

2 Este es un problema que fue tratado en detalle por el grupo de Mockus (1987) ¿Informática sin escritura? El problema para la educación. Cuadernos de Economía 10, segunda época, pp. 37-54. 
a las conversaciones escritas a través de redes sociales, y es conjugado como un verbo más del español.

A pesar de lo positivo que parezca, estas creaciones lingüísticas suelen ser constantemente criticadas por los docentes. Pues sostienen que son una agresión al idioma, ignorando que el cambio es persistente y que esto hace parte del mismo. Como asegura Daniel Cassany: "Sin duda, lo vernáculo ha existido siempre, aunque es más recientemente que ha incrementado su presencia, con el incremento de los contextos y los soportes y recursos para escribir". (Cassany, 2015)

Por su parte, algunos docentes, sobre todo los de edades avanzadas, se han visto en aprietos para poder comprender y aceptar esta nueva forma de escritura basada en abreviaturas que comprometen a veces solo a consonantes como una sola palabra, e incluso emojis, que son figuras expresivas que se usan al enviar mensajes y que expresan unos sentimientos y unas emociones precisas escogidas por el emisor, bien por ahorro lingüístico, o simplemente para la decoración estética del mensaje.

Si bien, algunos docentes han aceptado el reto y han intentado entender, adaptar y retomar la forma de escritura, otros, por el contrario, son reacios a estos cambios y han preferido seguir con su método tradicionalista de "enseñanza correcta de la lengua y el lenguaje" y ofuscar el intento de innovación de los más jóvenes. Emilia Ferreiro ilustra estos casos diciendo: "he escuchado docentes en posiciones extremas: algunos tratan de alertar sobre los peligros para la lengua (a través de la escritura) de estos desatinos juveniles; otros van al extremo opuesto y proponen espacios escolares para chatear, incluyendo a los profesores". (Ferreiro, 2006).

\section{Capacitación (Inmersión) NTIC}

Ante este panorama de incertidumbre, de desatinos y extremismo mediático de ambos bandos, tanto de educadores como de educandos, podría pensarse que para facilitar el aprendizaje y permitir el uso de las redes sociales y su aporte a la Lengua Castellana en los colegios y las aulas de clase, es necesario que los docentes tengan una capacitación-inmersión en el contexto de las NTIC, referidas principalmente al internet y las redes sociales, aunque también incluyendo métodos tecnológicos no necesariamente encaminados a las tecnologías web. Cuando un docente se ve cara a cara con las NTIC y desconoce su uso, puede identificarlo como un obstáculo para la continuación de la transmisión de la enseñanza. Quedan, entonces, dos opciones: dar la espalda al presente y futuro de sus estudiantes, o aceptarlas como un reto, aprender, bien sea por medio de una capacitación o por simple ejercicio empírico, y así generar un ambiente ameno en la escuela y el aula de clases. Espacios como el Campus Party ${ }^{3}$ deberían dedicar amplios espacios a la formación de docentes con el conocido bautizo tecnológico.

Las NTIC son imprescindibles en el proceso de enseñanza-aprendizaje: esta percepción por parte de algunos docentes hace referencia a su utilización sin un análisis previo sobre su utilidad en el contexto del aprendizaje, implicando de este modo subutilizarlas o, por el contrario,

3 Campus Party es una LAN Party, creada en España en 1997, que concentra aficionados a la informática, innovación, ciencia, creatividad, emprendimiento y ocio digital del mundo entero. En sus inicios se realizó en distintas localidades españolas como Málaga, Palma de Mallorca y Valencia; a partir de 2008 inició una expansión internacional con eventos en Brasil, Colombia, Ecuador, México, El Salvador, Reino Unido, Alemania y Costa Rica. Las actividades más comunes giran en torno a variados contenidos, entre los que están conferencias y talleres de astronomía, makers, desarrollo y programación, diseño, software libre, fotografía, emprendimiento, multimedia, drones, diseño de videojuegos, video, partidas de videojuegos multijugador. Además, está el intercambio de todo tipo de datos, información y de conocimientos en diversos campos temáticos relacionados con la temática del evento. Campus Party es reconocido como "el mayor evento de internet del mundo". Wikipedia, https://es.wikipedia.org/wiki/Campus_Party 
sobreutilizarlas de forma irresponsable, conduciendo a resultados no adecuados del proceso de enseñanza-aprendizaje. (Ávila, Riascos y Quintero, 2009).

\section{El internet, las redes sociales y los profes de la mano}

Primero debo precisar que las cosas pueden ser vistas como buenas o malas, según sean mostradas, entendidas y/o usadas. Constantemente oímos juicios de valor hacia situaciones que se hacen cotidianas y que suelen comprometer avances por el no entendimiento de las mismas. Es esto lo que ocurre con el internet y las redes sociales. Para los docentes que desconocen el uso y apoyo de las NTIC en el aula de clases, al momento de enfrentarse a ellas deberán dar un juicio de valor. Y sería decepcionante que a los estudiantes se les enseñe las redes sociales y el internet en general como algo negativo.

Pues bien, en el caso de la educación, y haciendo una comparación con los métodos educacionales en el mundo, como en las escuelas de Europa y Estados Unidos, vemos que el aliado número uno y de difícil oposición es el internet. Es inútil darle la espalda, debido a que hoy en día es vital en las personas. Un estudio realizado por eMarketer, publicado en la revista Clases de Periodismo, indicó que en el año 2016 habrá en el mundo cerca de dos mil millones de usuarios solamente de Smartphone (Cdperiodismo, 2016). Lo que también nos llevaría a pensar en que esa cantidad puede duplicarse frente a las personas que usan computadores y/o los nuevos aparatos de conexión digital, como las tablets, los Xbox interlinea, etc. De esta manera evidenciamos la necesidad de las personas al estar en constante conexión con el mundo del internet, la información inmediata y eficaz, y la rápida y efectiva comunicación a larga distancia.

En Colombia, Argentina y los demás países de América Latina ya se han intentado incursionar las NTIC en las escuelas. Sin embargo, han sido programas piloto que, de la mano con los gobiernos y los entes encargados de las NTIC, han puesto a prueba diferentes proyectos, de cuyos resultados no ha sido posible encontrar publicaciones o comentarios al respecto, más que felicitaciones y títulos de periódicos donde se documenta y registra los inicios de dichos programas, mas no la continuación de los mismos.

El ejercicio de entendimiento y aceptación de las NTIC como apoyo para las clases por parte de los docentes va a ser un proceso paulatino, desde luego. Para ello, toda la comunidad deberá apoyar y conocer las iniciativas tecnológicas. Y esto bien lo ha sabido liderar el Ministerio de Tecnologías de la Información y las Comunicaciones de Colombia (MINTIC), pues está liderando un proyecto titulado Plan Vive Digital Para la Gente 2014 - 2018, que, como lo reza en su página web, "busca reducir la pobreza, generar empleo, y desarrollar soluciones para los problemas de los colombianos, a través del uso estratégico de la tecnología".

Este proyecto trabaja a partir de cuatro pilares que son: Empleo, Ciudad y Región, Educación y Emprendimiento, y Gobierno Digital. Cabe resaltar que también ha buscado llevar a los más recónditos lugares del país implementos tecnológicos como tablets, computadores, video beam, etc., y ha buscado establecer una óptima conexión de internet en los establecimientos educativos, las ciudades y los pueblos en general, para facilitar las comunicaciones en línea y a distancia. Quizá parezca un esfuerzo paupérrimo, pero no dejan de ser pequeños pasos del gobierno colombiano por reconocer los espacios tecnológicos como urgentes frente a la sociedad actual, sobre todo en las comunidades con jóvenes en situación de escolaridad.

De igual forma, los grupos de investigación de las Universidades y sus correspondientes semilleros de investigación que incluyen el trabajo con las NTIC también han hecho su aporte, creando material didáctico que impulsa a los estudiantes a desarrollar sus conocimientos, por medio de programas didácticos, que no necesitan conexión de red, y que sirven de soporte para las clases de todas las áreas. Por ejemplo, existen programas de bibliotecas ligeras, que incluyen un manual-taller para 
trabajar obras literarias, información biográfica del autor o los autores, ubicación del contexto de las obras y juegos ilustrados que acompañen al educando a relacionarse de forma amena a los trabajos literarios en la escuela y en la casa. Un trabajo fuerte desde la academia es fortalecer los programas de ingeniería de sistemas, ingeniería de software, los cursos de programación, las plataformas digitales con el fin de estimular y fortalecer la sensibilización hacia el mundo digital. Una de las publicaciones más destacadas en este campo, Journal Computers in the Schools. Interdisciplinary Journal of Practice, Theory, and Applied Research, debería ser objeto de análisis no solo para implementar, previa adecuación cultural, las propuestas pedagógicas en el mundo digital sino también para proponer modelos conceptuales alternativos y nuevos métodos de trabajo.

\section{Apps educativas no nacidas con ese fin}

El mundo del internet constantemente se recrea con innovadoras aplicaciones y redes sociales con un sinfín de usos, desde los más educativos y útiles, como las más extrañas con utilidades fuera de lo convencional. Los dispositivos móviles, como las tablets y los teléfonos celulares Smartphone que cuentan con un sistema operativo Android, Windows Phone o Iphone pueden usarse para descargar las aplicaciones que el usuario requiera o considere necesarias para su quehacer diario. Es por eso que se encuentran personas que frecuentemente instalan aplicaciones como Diccionarios, Traductores, y las redes sociales en general. Otras personas usan aplicaciones de entretenimiento un poco más sofisticadas, como plataformas musicales, editores de fotos con filtros pro, y aplicaciones para ver películas en línea con síntesis de la misma incluida.

Estas aplicaciones no son ajenas a la realidad de la juventud, que tiene a su disposición todo este mundo cibernético, con la posibilidad de hacer uso de todas estas plataformas. Y cada vez son más las juventudes que usan redes sociales frecuentemente, tales como Instagram, Facebook, Twitter, Whatsapp, Messenger, Tumblr, Vk, Youtube, Flirck, Imgur y Snappchat, por mencionar algunos.

El atractivo de Internet para los jóvenes es que se caracteriza por la respuesta rápida, las recompensas inmediatas, la interactividad y las múltiples ventanas con diferentes actividades. El uso es positivo, siempre que no se dejen de lado el resto de las actividades propias de una vida normal (estudiar, hacer deporte, ir al cine, salir con los amigos o relacionarse con la familia). (E Echeburúa, P De Corral, 2010)

Pero no todas las aplicaciones móviles responden al ocio, pues existen otras más que, aunque nacieron con la intención de disfrutar a sus usuarios y distraerlos del mundo real, han sido usadas también para el trabajo educativo. En el caso de los espacios literarios, existen un sinfín de plataformas dedicadas a la libre creación y publicación de textos. Algunas de las más populares son Wattpad y Sttorybox. También hay aplicaciones que ayudan a aprender lenguas extranjeras, como es el caso de la más popular en este enfoque: Duolingo. $\mathrm{Y}$ en fin, se puede encontrar aplicaciones para aprender matemáticas, de enfoques científicos y muchos más, que incentiven las capacidades del aprendizaje.

Es esto lo que quiero resaltar de las plataformas virtuales, vistas como ayudas externas del sistema educativo, pero vinculadas discretamente en los procesos de aprendizaje de los niños y jóvenes. Pues, cuando el docente conoce y entiende la utilidad de las aplicaciones web, podrá usarlas para generar un interés didáctico de sus clases, de manera interactiva y entretenida ${ }^{4}$.

Es así que el docente, en lugar de ignorar y darle la espalda a estos espacios, deberá tomarlos como ayuda para sus clases. Lo importante aquí es aceptar que existen, que son una realidad y que no puede evitar que sus estudiantes hagan

4 Chang Liu (2012) 
uso de ellas. Desde luego que dispondrá de espacios para su trabajo, que, preferiblemente, serán por fuera del aula de clase. De manera que los espacios libres de los estudiantes puedan ser ocupados inconscientemente por juegos y talleres, en donde también hagan uso de su intelecto y desarrollen habilidades que les permite la web, demostrando ser un soporte para las clases y la escuela.

En conclusión, debemos entender que las NTIC, la web, las redes sociales y en general la internet son una realidad que ha pasado a tornar parte de la cotidianidad del mundo. Cualquier persona, sin importar su sexo, idioma, nacionalidad, edad o raza, puede hacer uso de estas. Y su inmersión en los espacios educativos, escuelas y aulas de clase, más que una realidad, es una necesidad. Por eso los docentes no deben desconocerlas, satanizarlas o darles la espalda, pues, en lugar de alivianar las cosas, estarían dando un retroceso en el avance educativo y eso debilitaría enormemente el desarrollo en la región, país, el continente $\mathrm{y}$, más importante aún, el estudiante. Los docentes deben saber usar la NTIC a su favor, proponerle trabajo a sus estudiantes por medio de estas, innovar, renovar, refrescar y encontrar de qué forma puede apoyarse en redes sociales populares como Facebook o Twitter, inventarse juegos y lograr el tan anhelado interés de sus estudiantes por la materia que ofrezca, de manera que el trabajo docente, el aula de clases y la escuela ya no sean vistos como un castigo, sino como una forma esencial de aprendizaje brillante, efectivo y llamativo para el educando.

\section{Referencias bibliográficas}

Vottero, B. (2007). Tensiones entre Escuela y Tecnologías (o el jardín de senderos que se bifurcan). Claro Oscuro: Villa María.

Ferreiro, E. (2006). Nuevas tecnologías y escritura. Reflexiones pedagógicas: Ciudad de México.

Cassany, D. (2015) Las ortografías en Internet: exploración, datos y reflexiones, Barcelona: Universitat Pompeu Fabra.

Ávila, Riascos y Quintero (2009). Las TIC en el aula: percepciones de los profesores universitarios. Bogotá: Universidad de la Sabana.

Barreras, R. (2016) 7 beneficios de la educación virtual. Bogotá: El nuevo siglo.

CDPeriodismo (2016) En 2016 habrá dos mil millones de usuarios de smartphones en el mundo. Clases de Periodismo: Lima. Recuperado de:http://www.clasesdeperiodismo. com/2014/12/23/en-2016-habra-dos-milmillones-de-usuarios-de-smartphones-en-elmundo/

El Plan Vive Digital. (2014-2018). Vive Digital para la gente. Bogotá: MINTIC. Recuperado de http://www.mintic.gov.co/portal/vivedigital

E Echeburúa, P De Corral. (2010) Adicción a las nuevas tecnologías y a las redes sociales en jóvenes: un nuevo reto. Red de Revistas Científicas de América Latina y el Caribe, España y Portugal: España.

Chang, Liu (2012). IERI Procedia 2, 247- 252 


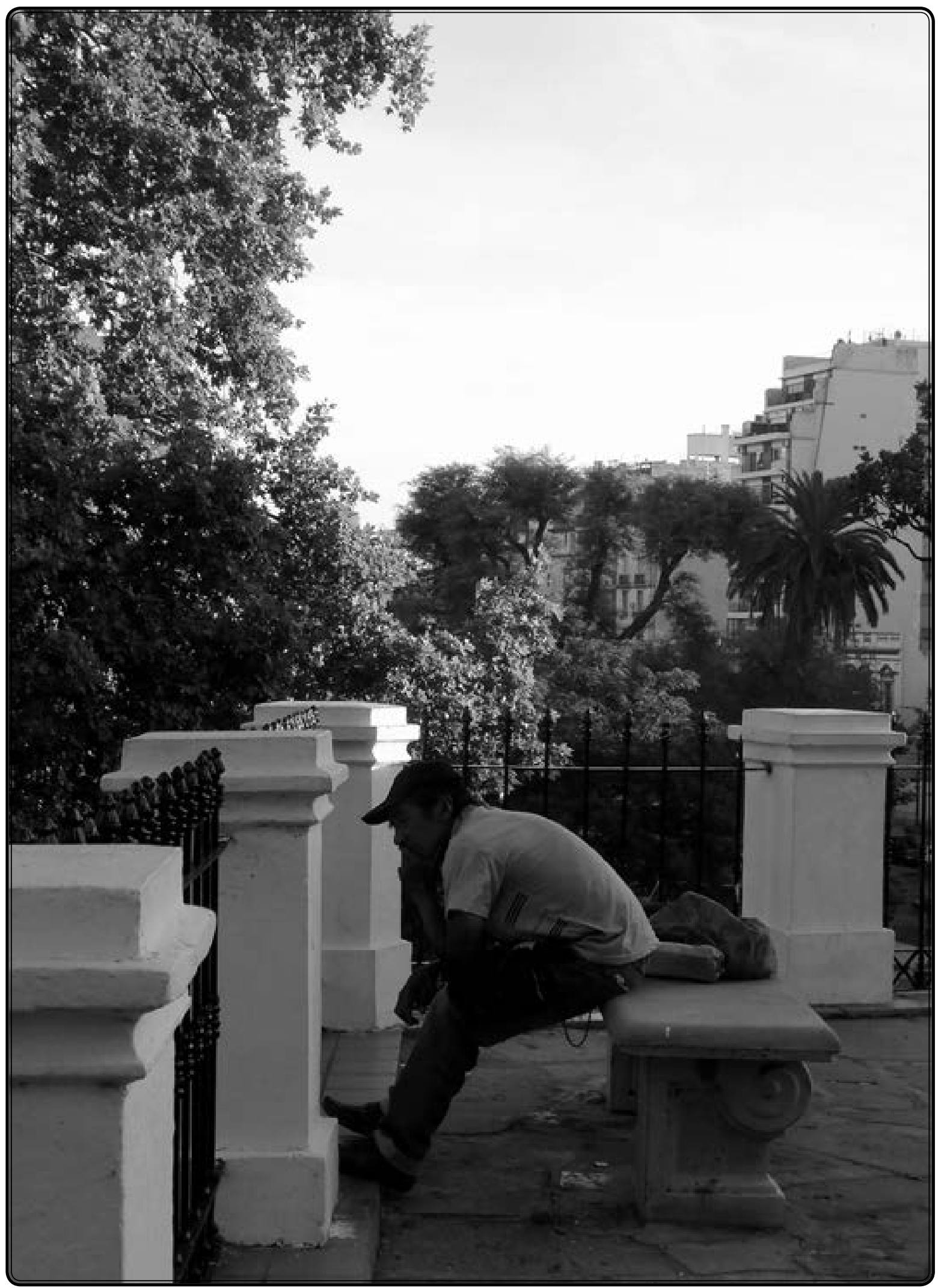

¿Por dónde comenzar? ¿Cuál es el camino? 